kidney cancer (HR $1.60[95 \% \mathrm{Cl} 1.15-2.23])$, sarcoma (HR $2.14[95 \% \mathrm{Cl} 1.41-$ 3.24]), acute-leukemia (HR 1.81 [95\% $\mathrm{Cl} 1.06-3.07]$ ), chronic-leukemia (HR $1.82[95 \% \mathrm{Cl} 1.19-2.77]$ ), Hodgkin's lymphoma (HR $2.42[95 \% \mathrm{Cl} 1.12-5.20]$ ), non-Hodgkin's lymphoma (HR $1.66[95 \% \mathrm{Cl} 1.21-2.29])$ and multiple myeloma (HR $2.40[95 \% \mathrm{Cl}$ 1.63-3.53) (Table 1). The time (mean [months] \pm SD) to the diagnosis of any malignancy was significantly shorter in GCA patients (48.6 \pm $41.3)$ compared to controls $(58.1 \pm 43.6 ; p<0.001)$.

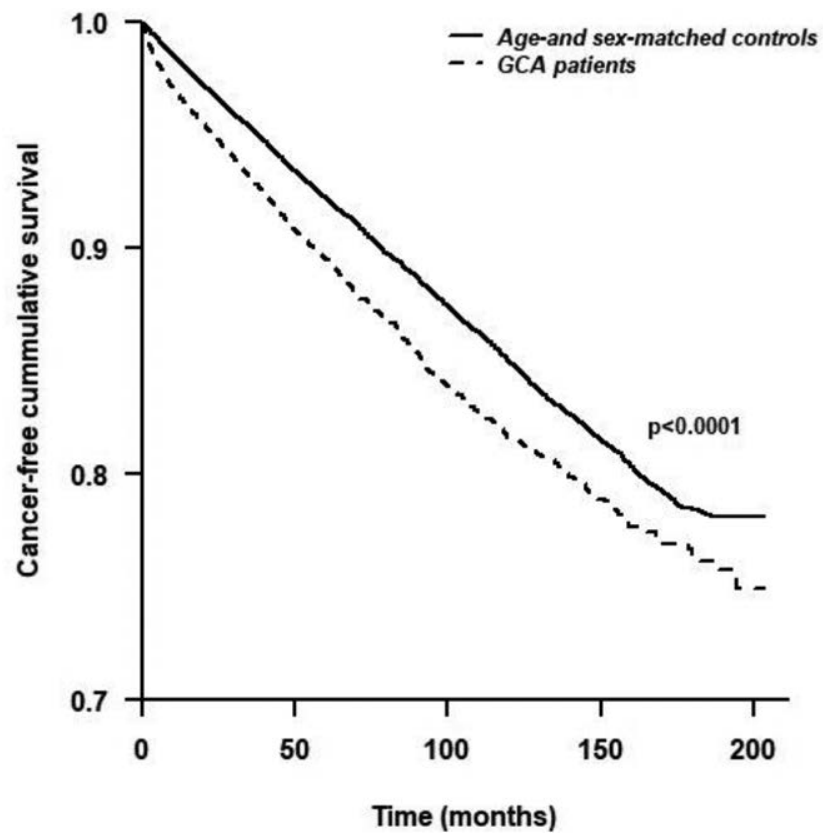

Figure 1. Kaplan-Meier cancer-free survival curve

Conclusion: GCA patients are at increased risk for sarcoma, kidney cancer, hematological malignancies and overall malignancies compared to age-and-sex matched controls from the general population.

References:

[1] Watad A, McGonagle D, Bragazzi NL, Tiosano S, Comaneshter D, Shoenfeld $\mathrm{Y}$, et al. Autoantibody status in systemic sclerosis patients defines both cancer risk and survival with ANA negativity in cases with concomitant cancer having a worse survival. Oncoimmunology 2019;8:e1588084.

[2] Ben-Shabat N, Tiosano S, Shovman O, Comaneshter D, Shoenfeld Y, Cohen $A D$, et al. Mortality among patients with giant-cell arteritis: A large-scale population-based cohort study. J Rheumatol 2019;

Disclosure of Interests: None declared

DOI: 10.1136/annrheumdis-2020-eular.6710

\section{OP0144 EFFECT OF TOCILIZUMAB ON VASCULAR INFLAMMATION BY 18F-FLUORODEOXYGLUCOSE POSITRON EMISSION TOMOGRAPHY: A PROSPECTIVE, LONGITUDINAL STUDY}

K. A. Quinn ${ }^{1,2}$, H. Dashora ${ }^{1}$, M. Ahlman ${ }^{1}$, P. Grayson ${ }^{1} .{ }^{1}$ National Institutes of Health, Bethesda, United States of America; ${ }^{2}$ MedStar Georgetown University Hospital, Washington, DC, United States of America

Background: Two randomized controlled trials have demonstrated clinical efficacy of tocilizumab for the treatment of giant cell arteritis $(G C A)(1,2)$. In these trials, clinical and laboratory measures were used to define the outcome measures. The direct effect of tocilizumab on vascular inflammation remains poorly characterized. Objectives: To prospectively evaluate vascular inflammation as measured by ${ }^{18} \mathrm{~F}$-fluorodeoxyglucose (FDG) positron emission tomography (PET) in a longitudinal cohort of patients with GCA treated with tocilizumab over a several year follow-up period.

Methods: Patients with GCA were recruited into a prospective, observational cohort. All patients fulfilled modified 1990 American College of Rheumatology (ACR) Classification Criteria for GCA. All patients underwent FDG-PET computed tomography (CT) prior to initiation of tocilizumab. A single reader reviewed all PET scans, blinded to clinical data. Qualitative assessment of FDG uptake relative to liver uptake by visual assessment (scale 0-3) was assessed in 9 arterial territories. A summary score, PET Vascular Activity Score (PETVAS), was calculated (scale 0-27).
Patients underwent imaging at 6-12 month intervals per a standardized imaging protocol. In a subset of patients in whom tocilizumab was discontinued due to established remission, a repeat FDG-PET scan was obtained within 6 months of drug discontinuation.

Change in PET activity over time was measured by linear regression. PET activity during established remission was compared to PET activity after discontinuation of tocilizumab. For some patients, tocilizumab was added to the existing treatment regimen without a substantive change in concomitant glucocorticoid dose. In a secondary analysis, patients were stratified by prednisone dosing (high dose prednisone $>10 \mathrm{mg} /$ day prednisone, low dose prednisone $\leq 10 \mathrm{mg} /$ day prednisone during the imaging interval) to determine if tocilizumab had an effect on vascular inflammation independent of glucocorticoids.

Results: 22 patients were included in the study. All patients had clinically active disease at baseline with median baseline PETVAS 24.5 (23-27). There was a significant reduction in PETVAS over 2 years follow up ( $p<0.01$ for linear trend) (Figure). Of note, there was continued progressive improvement in PETVAS in both year 1 and year 2 of treatment. Eight patients received concomitant high dose glucocorticoids and 14 patients remained on low dose glucocorticoids with the addition of tocilizumab. In patients who only received low dose prednisone, significant reduction in PETVAS was still observed with addition of tocilizumab (PET VAS 25.5 to $19.5, p=0.04$ ). In a subset of 5 patients who discontinued tocilizumab due to established remission [median PETVAS 19 (17.5-22) at time of remission], a repeat FDG-PET scan within 6 months after treatment discontinuation showed worsening PET activity in 4 out of 5 patients [median PETVAS 23 (20-23)]. Two of these patients subsequently experienced a clinical disease relapse.

Conclusion: Tocilizumab was associated with improved vascular inflammation as assessed by FDG-PET. There was continued improvement of vascular inflammation at both year 1 and year 2 of treatment, and early evidence suggests a rebound of vascular inflammation when tocilizumab was discontinued. These data provide rationale for long-term tocilizumab therapy in patients with GCA and for consideration of FDG-PET as an outcome measure in future clinical trials. References:

[1] Stone JH, et al. N Engl J Med 2017;377:317-28.

[2] Villiger PM, et al. Lancet 2016;387:1921-7.

PET Activity vs. Elapsed Time on Tocilizumab

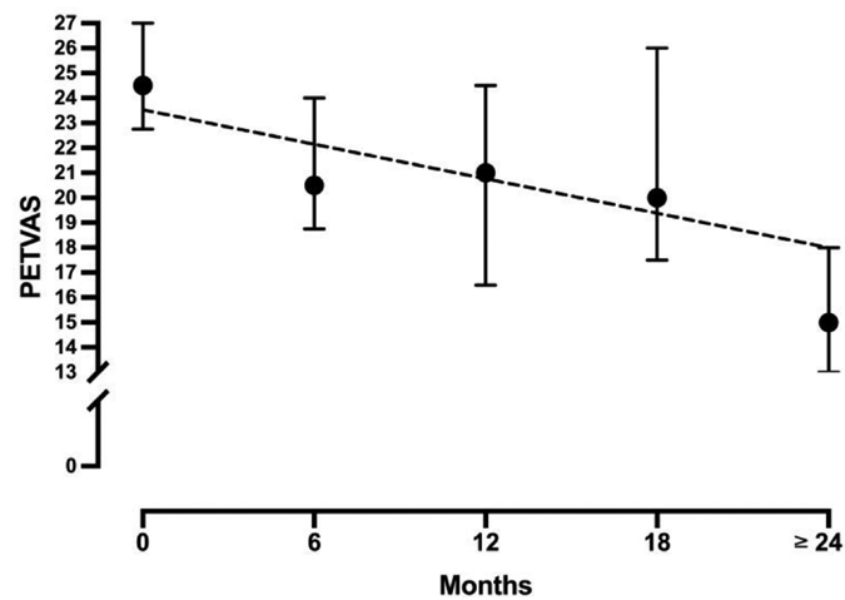

Figure. $X X X$

Disclosure of Interests: None declared DOI: 10.1136/annrheumdis-2020-eular.2840

\section{OP0145 \\ MALIGNANCY IN ANCA-ASSOCIATED VASCULITIS AND POLYARTERITIS NODOSA: AN AUSTRALIAN POPULATION-BASED STUDY}

J. Tieu ${ }^{1,2,3,4}$ S. Lester ${ }^{1,2}$, W. Raymond ${ }^{5}$, H. Keen ${ }^{5,6}$, C. Hill ${ }^{1,2,4}$, J. Nossent ${ }^{5,7}$. ${ }^{1}$ Faculty of Health and Medical Sciences, University of Adelaide, Adelaide, Australia; ${ }^{2}$ Basil Hetzel Institute, Adelaide, Australia; ${ }^{3}$ Lyell McEwin Hospital, Adelaide, Australia; ${ }^{4}$ The Queen Elizabeth Hospital, Adelaide, Australia;

${ }^{5}$ University of Western Australia, Rheumatology, Perth, Australia; ${ }^{6}$ Fiona Stanley Hospital, Rheumatology Dept, Perth, Australia; ${ }^{7}$ Sir Charles Gairdner Hospital, Rheumatology Dept, Perth, Australia

Background: The increased risk of malignancy in patients with ANCA-associated vasculitis (AAV) and polyarteritis nodosa (PAN) has been attributed to late 